Research Paper

\title{
The metabonomics study of P-selectin glycoprotein ligand-1 (PSGL-1) deficiency inhibiting the progression of atherosclerosis in $\mathrm{LDLR}^{-/-}$mice
}

\author{
Binglin Li, Xin Lu, Jia Wang, Xiaodong He, Quliang Gu, Lijing Wang ${ }^{\bowtie}$, Yongxia Yang ${ }^{\bowtie}$ \\ Vascular Biology Research Institute, School of Basic Course, Guangdong Pharmaceutical University, Guangzhou, 510006, PR China \\ $\triangle$ Corresponding authors: Prof. Yongxia Yang: School of Basic Course, Guangdong Pharmaceutical University, Guangzhou; 510006, PR China; Tel: \\ +86-(0)20-39352197, Fax: +86-(0)20-39352186, Email: yongxiayang2017@163.com Prof. Lijing Wang, Vascular Biology Research Institute, School of Basic Course, \\ Guangdong Pharmaceutical University, Guangzhou; 510006, PR China; Tel: +86-(0)20-39352126, Fax: +86-(0)20-39352126, Email: ljwang2017@163.com \\ (C) Ivyspring International Publisher. This is an open access article distributed under the terms of the Creative Commons Attribution (CC BY-NC) license \\ (https://creativecommons.org/licenses/by-nc/4.0/). See http://ivyspring.com/terms for full terms and conditions.
}

Received: 2017.09.28; Accepted: 2017.11.17; Published: 2018.01.01

\begin{abstract}
Atherosclerosis (AS) is a multi-factorial chronic disease commonly associated with the mechanisms of metabolism disorder, endothelial dysfunction and chronic inflammation. AS an inflammatory molecule, p-selectin glycoprotein ligand-1 (PSGL-1) played an important role in the inflammatory process of atherogenesis involving the recruitment of leukocyte and transmitting signals to activate leukocyte during the adhesion process. So far, there has been little study regarding the effects of PSGL-1 on AS progression and the metabolic regulation. In this report, we studied the effect of PSGL-1 deficiency on the formation and progression of AS and the metabolic regulation by use of $\mathrm{LDLR}^{-1 /}$, PSGL- ${ }^{-1 /}$ transgenic mice based on metabonomics. It was found that the PSGL-1 deficiency reduced the atherosclerotic plaque area, inflammatory cells infiltration and fiber hyperplasia during the AS development. The serum metabonomics study showed that the LDLR ${ }^{-1}, \mathrm{PSGL}^{-1 /-}$ mice had higher levels of HDL, valine, acetate, pyruvate, choline, PC, GPC and glycine, and lower levels of LDL+VLDL and lactate at the early stage of atherosclerosis, while lactate, citrate and glutamine showed statistical significance at the late stage of atherosclerosis. These results showed that the PSGL-1 deficiency inhibited the AS progression and regulated glucose metabolism, lipid metabolism, amino acid and phospholipid metabolism in LDLR ${ }^{-1-}$ mice.
\end{abstract}

Key words: Atherosclerosis; PSGL-1; ${ }^{1} \mathrm{H}$ NMR; metabonomics; LDLR $\%$ mice.

\section{Introduction}

Atherosclerosis (AS), as a chronic disease of medium and large arteries [1], is the leading factor of cardiovascular diseases [2-3]. The vast research efforts that were made to disclose the mechanisms of atherosclerosis have been done. At present, the basic progression of AS lesion includes arterial endometrial injury, lipid deposition and inflammatory response, and then the atherosclerotic plaque and fibrosis hyperplasia are formatted, causing the vessel wall hardening and arterial lumen narrowing [4-6]. It is well known that the mechanisms of AS include the metabolism disorder, endothelial dysfunction and chronic inflammation, et al [7-10]. And dyslipidemia, hypertension and diabetes are the major risk factors for the development of atherosclerosis [11-13].

AS an important inflammatory molecule, p-selectin glycoprotein ligand-1 (PSGL-1) is mainly expressed on all types of leukocytes and is the main glycoprotein ligand of selectin [14-15]. PSGL-1 not only plays an important part in the recruitment of leukocyte during the adhesion process, but also acts as a signaling molecule transmitting signals to activate leukocyte [16]. The previous study showed that PSGL-1 deficiency could inhibit the adhesion of endothelial cells and leukocytes through cytokines, and reduced the atherosclerosis in ApoE-/- mice [17]. 
It was reported that PSGL-1 play pivotal roles in the inflammatory process of atherogenesis [18]. So far, there has been little study regarding the metabolic regulation effect of PSGL-1 on AS.

LDLR $\%$ mice is a classic animal model of atherosclerosis. The atherosclerosis pathogenesis in $\mathrm{LDLR}^{-/-}$mice was similar with the clinical lesions, which has been widely used in the study of atherosclerosis. Study has reported that the aorta exhibited gross atheroma and the aortic valve leaflets were thickened by cholesterol-laden macrophages in the LDLR $\%$ mice with a high-fat diet [19]. Additionally, the western diet-induced atherosclerosis progression in LDLR $\%$ mice was accompanied by metabolic changes, such as the disorders of cholesterol homeostasis, and the alterations of amino acids, proteins and gut microbiota [20].

The metabonomics could explore the metabolic response of organisms induced by intrinsic and external factors stimuli [21-22]. The analytical techniques in metabonomics include HPLC-MS and NMR, etc [23-24]. The metabonomics has broad applications in disease diagnosis, pathology and toxicology researches [25-26], due to its main advantages of non-destruction, simplicity of sample preparation, high reproducibility and dynamic acquisition [27-29]. It was indicated that ${ }^{1} \mathrm{H}$ NMR-based metabonomics is an effective tool for monitoring the process of AS, demonstrating the time-related metabolic changes of multiple biological matrices during the occurrence and development of AS [30]. Furthermore, studies showed that the multiple biochemical disorders including energy metabolism, fatty acid and lipid metabolism were found in the progression of atherosclerosis [20,31].

Although PSGL-1 plays an important part in AS, the effect of PSGL-1 on the AS development and the metabolic regulation in LDLR $/$ - mice has not been reported. In this report, we studied the effects of PSGL-1 deficiency on the formation and progression of AS and the metabolic regulation by use of LDLR $^{-/,}, \mathrm{PSGL}_{-1}{ }^{-/}$transgenic mice based on metabonomics, which provided a new basis for the prevention and treatment of clinical AS.

\section{Materials and Methods}

\section{Chemicals}

Methanol (HPLC/PREP) and acetonitrile (HPLC/ACS) were purchased from J\&K Scientific LTD (Beijing, China). Deuterium oxide $\left(\mathrm{D}_{2} \mathrm{O}\right)$ was purchased from Qingdao Teng Long Technology Co., LTD (Qingdao, China). Disodium hydrogen phosphate $\left(\mathrm{Na}_{2} \mathrm{HPO}_{4}\right)$ and sodium dihydrogen phosphate $\left(\mathrm{NaH}_{2} \mathrm{PO}_{4}\right)$ are domestic analytical reagents. The Oil Red O (O 0625-25G) was obtained from Sigma-Aldrich in China.

\section{Model and Sample collection}

LDLR-/- ( B6. 129S7-Ldlrtm1Her /J) and PSGL-1/- ( B6. Cg-Selplgtm ${ }^{1 F u r} / J$ ) mice were purchased from Laboratory of Jackson (Bar Harbor, ME). The experimental mice were raised in the SPF environment where temperature and humidity were kept in a suitable range of $22-26{ }^{\circ} \mathrm{C}$ and $40-60 \%$ with a normal dark-light cycle. LDLR/-- mice were crossed to PSGL-1 $\%$ mice to generate $\mathrm{LDLR}^{+/-}, \mathrm{PSGL}-1^{+/-}$mice, which were then mated to generate LDLR $/$,PSGL-1 1 (as PSGL-1 deficiency model) and LDLR-/,,PSGL-1//+ mice (LDLR-/-mice, as control) used in this study. This research was agreed by the Ethics Review Committee of Guangdong Pharmaceutical University, China.

The 8 weeks-old LDLR $/$-,PSGL-1//+ $(n=37)$ mice were randomly assigned to four groups, i.e. a group at the 8 weeks $(n=8)$, and three groups continuously given a high-fat diet for another 8 weeks $(n=9), 12$ weeks $(n=10)$ and 16 weeks $(n=10)$, respectively. The same grouping was performed in LDLR $\%$,PSGL-1 1 $(n=36(8,8,10,10))$ mice. The high-fat diet (D12492) was obtained from the Animal Center of Guangdong (Guangdong, China). For convenience, the four groups in each kind of mice were denoted as $8 \mathrm{w}$ (only normal diet), $8+8 \mathrm{w}$ (high-fat diet for 8 weeks), $8+12 \mathrm{w}$ (high-fat diet for 12 weeks) and $8+16 \mathrm{w}$ (high-fat diet for 16 weeks). The control groups in LDLR $^{-/,}$, PSGL-1 ${ }^{+/+}$and LDLR $/$-,PSGL-1 $1 /$ models were sacrificed for serum and tissue (aorta and heart) collection at the mentioned-above time points. The serum and tissue samples were divided into two parts, one for biochemical assays or pathological examination, and another one were stored at $-80^{\circ} \mathrm{C}$ for NMR analysis.

\section{Biochemistry and histopathological examination}

Clinical chemistry analyses including TG, TC, LDL-C and HDL-C were assessed by use of a chemical analysis kit (Biosino Biotech, China). Statistical analysis was performed for the data using SPSS (Version 16.0, USA).

For the atherosclerosis assessments, the dissected aortic tree was fixed in $4 \%$ solution of paraformaldehyde. Oil red $\mathrm{O}$ staining was performed for the aortic trees for 30-40 $\mathrm{min}$ and then were assimilated the background with $60 \%$ isopropyl alcohol. And the aortic roots were put in the molds of Tissue-Tek OCT and frozen at $-20{ }^{\circ} \mathrm{C}$ for slice and microscopic analysis. The stainings of Oil red $\mathrm{O}$ and hematoxylin counterstaining were performed for the 
collected slices (with thickness of $7 \mu \mathrm{m}$ ) to evaluate the lipid accumulations. The assessment of atherosclerosis was performed by the lesion area. In order to assess the inflammatory cells infiltration and fiber hyperplasia in aortic root, hematoxylin and Sirius red staining were performed for the slices.

\section{'H NMR Spectroscopy}

Serum sample was thawed and $300 \mu \mathrm{L}$ of serum was taken and mixed with $100 \mu \mathrm{L}$ of PBS $\left(\mathrm{Na}_{2} \mathrm{HPO}_{4} / \mathrm{NaH}_{2} \mathrm{PO}_{4} \quad 0.1 \mathrm{M}, \quad \mathrm{pH}=7.4\right)$ After centrifuging $\left(15 \mathrm{~min}, 4^{\circ} \mathrm{C}, 5000 \mathrm{rpm}\right), \mathrm{D}_{2} \mathrm{O}(100 \mu \mathrm{L})$ was mixed with the supernatant and then pipette into $5 \mathrm{~mm}$ NMR tubes.

NMR ${ }^{1} \mathrm{H}$ spectra of serum samples were collected on a $500 \mathrm{MHz}$ spectrometer (Avance III, Bruker, Inc.) at $298 \mathrm{~K}$. The spectra were recorded for each sample with CPMG (Carr-Purcel-Meiboom-Gill) pulse sequence (pulse delay-90-( $(-180-\tau)_{\mathrm{n}}$-collection) with water presaturation. The spectra were acquired with 128 transients, 32k data points and $20 \mathrm{ppm}$ spectral width. The recycle delay is 3 s. An exponential function with $0.3 \mathrm{~Hz}$ line-broadening was used during Fourier transformation. Chemical shift were referenced to the double peaks of lactate $(\delta 1.33)$. Peaks at $\delta$ 0.5-9.5 were integrated into buckets with 0.004 ppm width by use of the AMIX (V3.7.3, Bruker Biospin, Germany). The integral values at $\delta$ 4.50-5.20, $\delta 1.15-1.24$ and $\delta 3.55-3.57$ were set to be zero in order to remove the effects of water and diethyl-ether.

\section{Multivariate data analysis and statistical analysis}

The integral data of the spectrum was normalized before the multivariate data analysis. Pattern recognition analysis was performed with SIMCA-P software (V12.0, Umetrics, Sweden). AS an unsupervised analysis method, PCA was carried out to classify the samples based on their spectra data without any prior manipulation [32]. Then OPLS-DA was performed for the spectra data with unit-variance scaling, in order to screen the differential metabolites of each unit-group. The predictability and interpretability of the model can be monitored according to the values of $\mathrm{Q}^{2}$ and $\mathrm{R}^{2}$, respectively [33, 34]. The results of PCA and OPLS-DA are represented by the scores plots, where each point denotes a sample. The loadings plots were back-transformed and were shown in coefficient-coded form. The variables in loadings plots were colored with the correlation coefficients from OPLS-DA (such as red, blue) by use of a MATLAB programmer. The metabolites significance was further statistically analyzed by a Student's t test (V19.0, SPSS).

\section{Results}

\section{Histopathological assessment of atherosclerosis lesions}

Figure 1 presented a progressively histopathological evaluation in aorta of LDLR $^{-/-, P S G L-1 / /+}$ and LDLR ${ }^{-/-, P S G L-1 / /-~ m i c e . ~ I t ~ w a s ~}$ found that there were no aortic lesions in LDLR $^{-/,}$, PSGL-1 $1 /-$ and LDLR $^{-/,}, \mathrm{PSGL}^{-1 /++}$ mice at 8 weeks (Figure 1A). During the high-fat diet, the ratio of placental aortic plaque area to vascular area increased gradually with prolonged high-fat diets (Figure 1B, C). As the figure shown, the plaque began to appear at $8+8 \mathrm{w}$, but the percentage of lesion area has no statistical difference between the two kinds of mice. The aortic plaque areas continued to increase at $8+12 \mathrm{w}$ and $8+16 \mathrm{w}$. It was noted that the plaque areas in LDLR $/$-,PSGL-1 $\%$ mice was much more less than those in $\mathrm{LDLR}^{-/,}, \mathrm{PSGL}-1^{+/+}$mice with statistical significance.

Further histopathological examinations were performed for the aortic root lesions in LDLR $^{-} /$, ,PSGL-1 ${ }^{+/+}$and LDLR $/$-,PSGL-1 $1 /-$ mice at $8 \mathrm{w}$, $8+8 w, 8+12 w$ and $8+16 w$ in order to reveal the effect of PSGL-1 deficiency on atherosclerosis. The HE, Oil red $\mathrm{O}$ and Sirius red staining of aortic roots at 8 weeks presented a good preservation of aortic wall and valve morphology, which suggested there were no atherosclerotic lesions at 8 weeks only with normal chow (Figure 2A). After 8 weeks of high-fat diets, the HE staining demonstrated the small accumulations of foam cells and the inflammatory cells infiltration into the coronary sinuses walls, Oil red-stained sections showed the lipid plaque deposits, and Sirius red staining presented a slight amount of fibrous proliferation occurred around the accumulation of foam cells in two kinds of mice. Compared with the LDLR $^{-/,-P S G L-1+/+}$ mice, the above-mentioned histopathological changes were much lighter in the LDLR $1 /$,PSGL-1 $1 /$ mice at $8+8 \mathrm{w}$ (Figure $2 \mathrm{~B}$ ). With the progression of $\mathrm{AS}$, the atherosclerotic plaque area, inflammatory cells infiltration and fiber hyperplasia continued to aggravate at $8+12 \mathrm{w}$ (Figure 2C), while the histopathgological changes was significantly inhibited in LDLR-/,PSGL-1/- mice. At 8+16w, more massive atherosclerotic plaque deposits within the proximal aorta and a typical atherosclerotic profile appeared involving lipid-rich core and severe inflammatory cell infiltration in LDLR-/,,PSGL-1 ${ }^{+/+}$ mice. It was noted that the difference between the

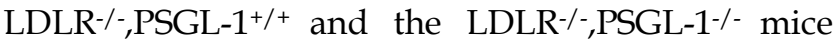
became obvious, which suggested that the deficiency of PSGL-1 inhibited the development and progression of atherosclerosis (Figure 2D). 
$\mathbf{A}$

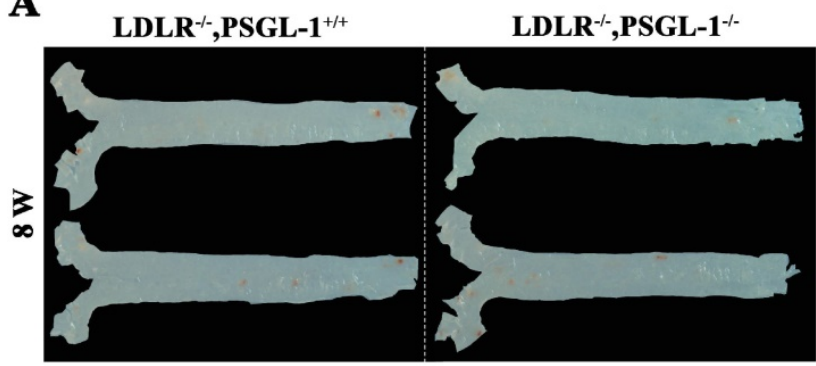

C

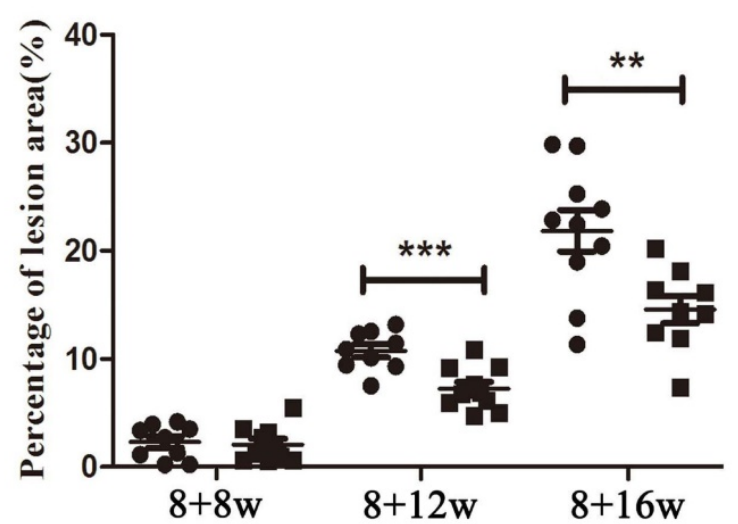

B
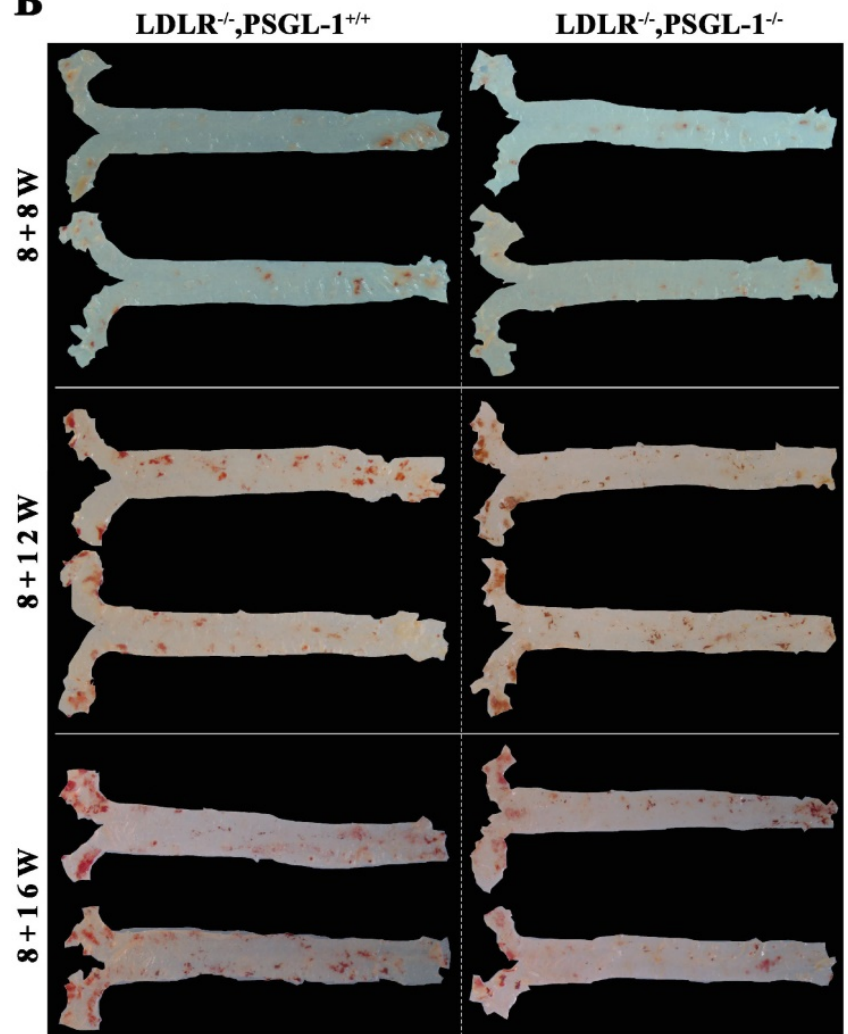

Figure 1. The Oil red $\mathrm{O}$ staining of aortic plaques. The oil-red staining of the aorta from LDLR-/,PSGL-1+/+ and LDLR-/-PSGL-1-/- mice at $8 \mathrm{w}(\mathrm{A}) ; 8+8 \mathrm{w}, 8+12 \mathrm{w}$, $8+16 \mathrm{w}(\mathrm{B})$ with statistical analysis results of the atherosclerotic plaque percentage (C). Note: * LDLR-/,PSGL-1-/- mice $(\boldsymbol{\square})$ vs. LDLR-/-,PSGL-1+/+ mice $(\bullet)$; **, P $<0.01$; $* * *, p<0.001$.

\section{Biochemical assessment of blood}

The biochemical results showed that TC, TG, HDL-C and LDL-C were at the normal levels at $8 \mathrm{w}$ (Table 1). With the progression of AS, we found that TC, TG and LDL-C up-regulated in LDLR $-/$, PSGL- $1^{+/+}$mice at $8+8 \mathrm{w}, 8+12 \mathrm{w}$ and $8+16 \mathrm{w}$. In contrast to the LDLR $/-$ mice, TC, TG and LDL-C decreased, while HDL-C increased in LDLR $/$-,PSGL-1/-mice, which showed the regulation effect of PSGL-1 deficiency on blood lipid metabolism.

\section{Metabolite profiling of serum samples}

Six representative serum ${ }^{1} \mathrm{H}$ CPMG spectra from LDLR $^{-} /$, PSGL- ${ }^{+/+}$and LDLR $^{-} /$, PSGL- $1 \%$ mice at $8+8 \mathrm{w}$ (Figure 3A, B), $8+12 \mathrm{w}$ (Figure 3C, D) and 8+16w (Figure $3 \mathrm{E}, \mathrm{F}$ ) were shown. The metabolites were assigned according to the literatures $[35,36]$ and our published data [31]. The serum ${ }^{1} \mathrm{H}$ NMR spectra were dominated by lipid, glucose, fatty acids and amino acids, etc. Visually, the serum metabolic profiles showed no obvious differences between these two groups of mice. Therefore, we performed pattern recognition analysis to disclose the metabolites differences in LDLR $^{-/,}$,PSGL-1 $\%$ and LDLR ${ }^{-/-, P S G L-1+/+}$ mice.

\section{Pattern recognition analysis}

The 3D scores plots of PCA showed the distributions of all serum samples at the $8 \mathrm{w}, 8+8 \mathrm{w}$, $8+12 \mathrm{w}$ and $8+16 \mathrm{w}$ in LDLR $^{-/,}, \mathrm{PSGL}-1^{+/+}$(Figure $4 \mathrm{~A}$ ) and LDLR $\%$-PSGL-1 $\%$ - mice (Figure $4 \mathrm{~B}$ ), respectively. Figure 4A showed the obvious classification among the four groups in LDLR-/,PSGL-1 ${ }^{+/+}$mice, in which the arrow denoted the trajectory of the distributions. The result revealed that there were distinct metabolic profiles during the AS progression. The similar samples distribution was found in LDLR $/$,, PSGL-1/mice, which demonstrated that the metabolic characteristics were closely correlated with the progression of atherosclerosis.

In order to obtain the metabolic changes induced by PSGL-1 deficiency, we performed the principal component analysis between LDLR $/ /$, PSGL-1 ${ }^{+/+}$and LDLR $\%$, $\mathrm{PSGL}-1 \%$ mice at the same time point. As shown in the figure $5 \mathrm{~A}$, there was no difference in the serum samples at $8 \mathrm{w}$. During the high-fat diet, the two kinds of mice had more and more separations (Figure 5B, C and D). These results showed that there were obvious metabolic difference between the two kinds of mice with the progression of AS. 

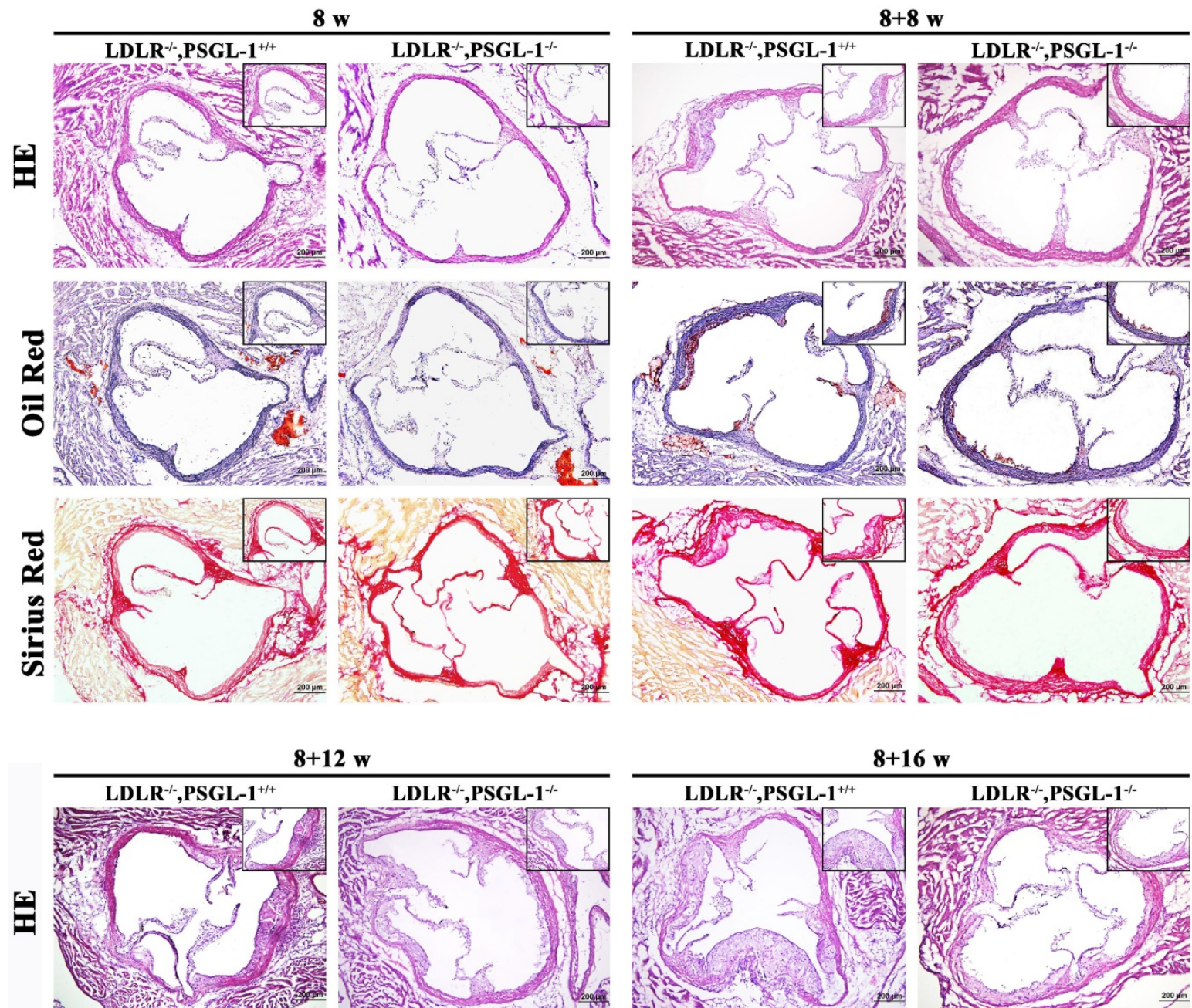

$8+12 \mathrm{w}$
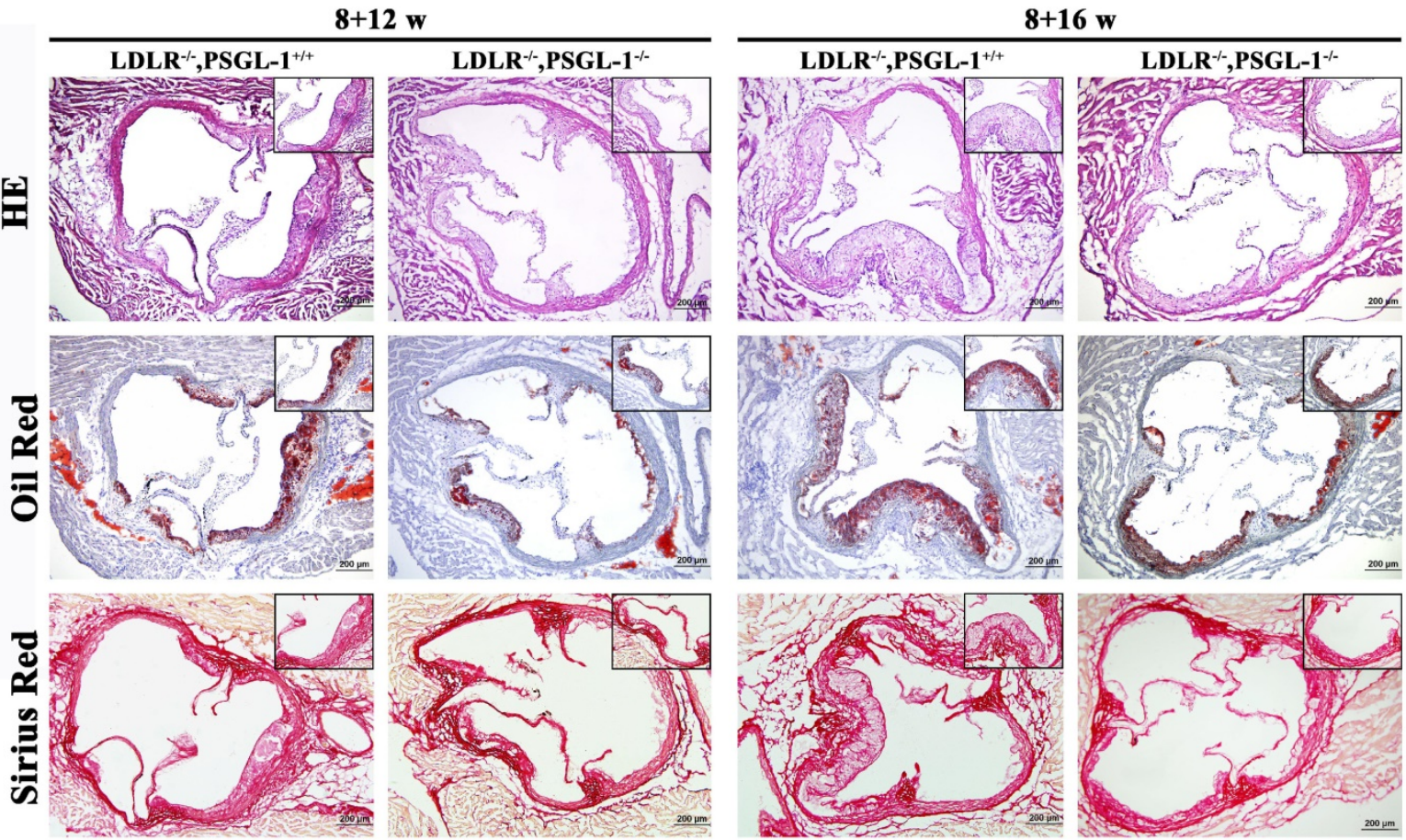

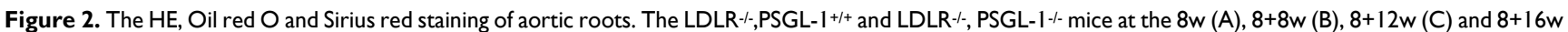
(D).

Table 1. Biochemical assessment of the LDLR ${ }^{-/}$, PSGL-1 $^{+/+}$and LDLR-/-PSGL-1/-- mice.

\begin{tabular}{|c|c|c|c|c|c|c|c|c|}
\hline Time & $8 w$ & & $8+8 w$ & & $8+12 w$ & & $8+16 w$ & \\
\hline & ${\mathrm{L}-/, \mathrm{P}^{+/+}+}^{-}$ & $\mathrm{L}-/, \mathrm{P}-/-$ & $\mathrm{L}^{-/,-\mathrm{P}^{+/+}}$ & $\mathrm{L}-/, \mathrm{P}-/-$ & ${\mathrm{L}-/, \mathrm{P}^{+/+}+}^{-}$ & $\mathrm{L}-/, \mathrm{P}-/-$ & $\mathrm{L}^{-/,-\mathrm{P}^{+/+}+}$ & $\mathrm{L}-/, \mathrm{P}-/--$ \\
\hline $\mathrm{TC}(\mathrm{mmol} / \mathrm{L})$ & $4.71 \pm 0.11$ & $5.41 \pm 0.33$ & $13.49 \pm 0.87$ & $11.60 \pm 1.09$ & $15.55 \pm 0.53$ & $12.65 \pm 0.55^{* *}$ & $19.87 \pm 1.07$ & $16.13 \pm 1.54^{*}$ \\
\hline $\mathrm{TG}(\mathrm{mmol} / \mathrm{L})$ & $1.77 \pm 0.09$ & $1.30 \pm 0.07$ & $2.71 \pm 0.17$ & $1.74 \pm 0.06^{* * *}$ & $2.96 \pm 0.36$ & $2.45 \pm 0.14$ & $2.36 \pm 0.27$ & $1.59 \pm 0.19^{*}$ \\
\hline HDL-c $(\mathrm{mmol} / \mathrm{L})$ & $1.19 \pm 0.16$ & $1.34 \pm 0.14$ & $1.96 \pm 0.08$ & $2.57 \pm 0.25^{*}$ & $2.05 \pm 0.09$ & $2.46 \pm 0.28^{*}$ & $2.56 \pm 0.27$ & $4.03 \pm 0.41^{*}$ \\
\hline LDL-c (mmol/L) & $1.56 \pm 0.18$ & $1.38 \pm 0.14$ & $7.88 \pm 0.49$ & $5.57 \pm 0.31^{* *}$ & $11.76 \pm 1.04$ & $9.11 \pm 0.38^{*}$ & $15.99 \pm 0.69$ & $14.47 \pm 1.09$ \\
\hline
\end{tabular}

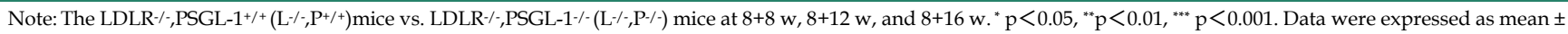
SEM. 


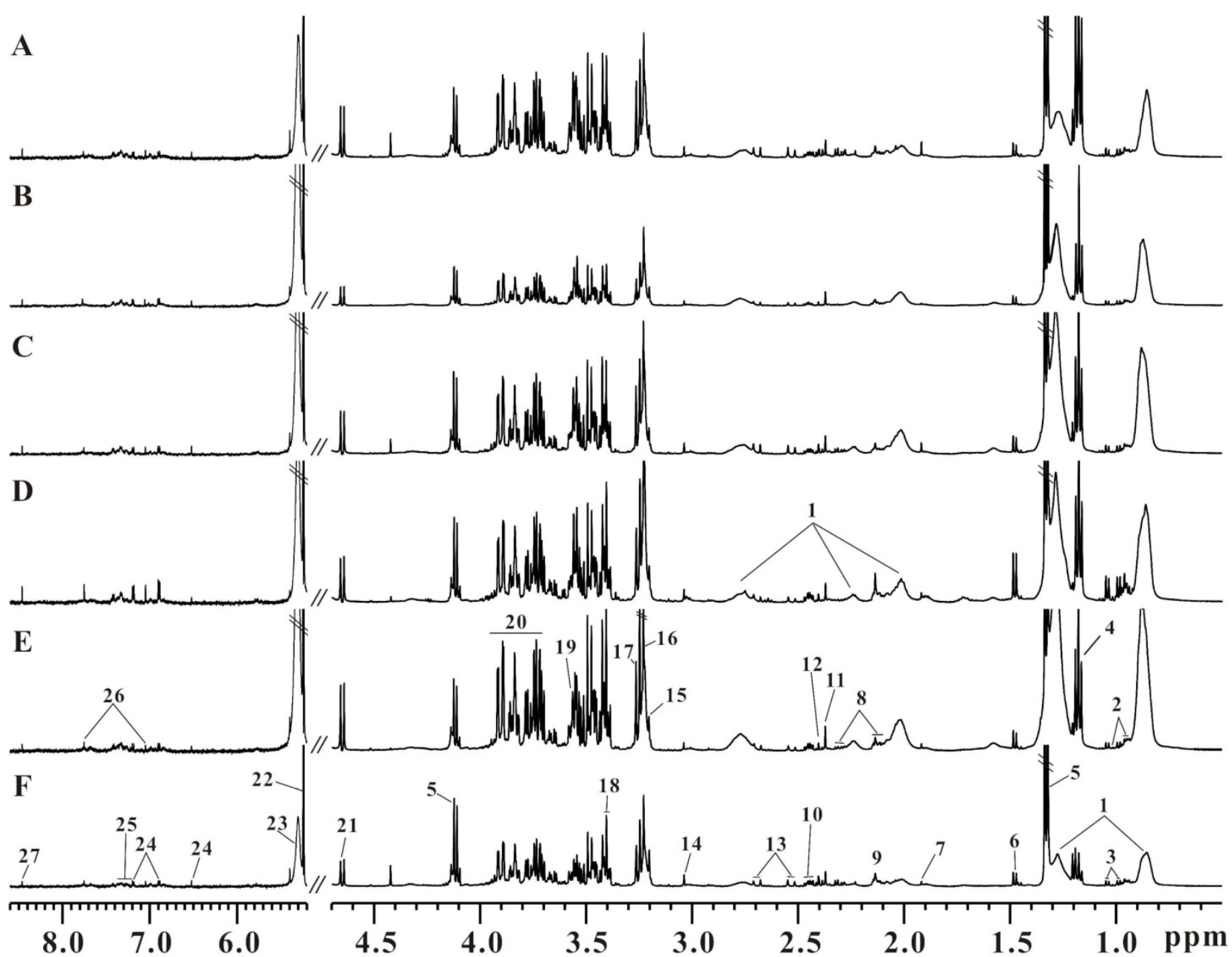

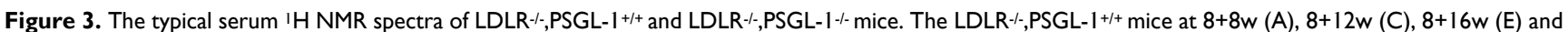
the LDLR-/-PSGL-1-/- mice at 8+8w (B), 8+12w (D), 8+16w (F). Note: 1.LDL and VLDL; 2.leucine and isoleucine; 3.valine; 4.aether; 5.lactate; 6.alanine; 7.acetate; 8.glutamate; 9.methionine; 10.glutamine; 11.pyruvate; 12.succinate; 13.citrate; 14.creatine; 15.choline; 16.phosphocholine (PC) and glycerophosphosphocholine (GPC); 17.betaine; 18.taurine; 19.glycine; 20.glucose and amino acids; 21. 3 -glucose; 22.a-glucose; 23.unsaturated fatty acids; 24.tyrosine; 25.phenylalanine; 26.histidine; 27.formate.
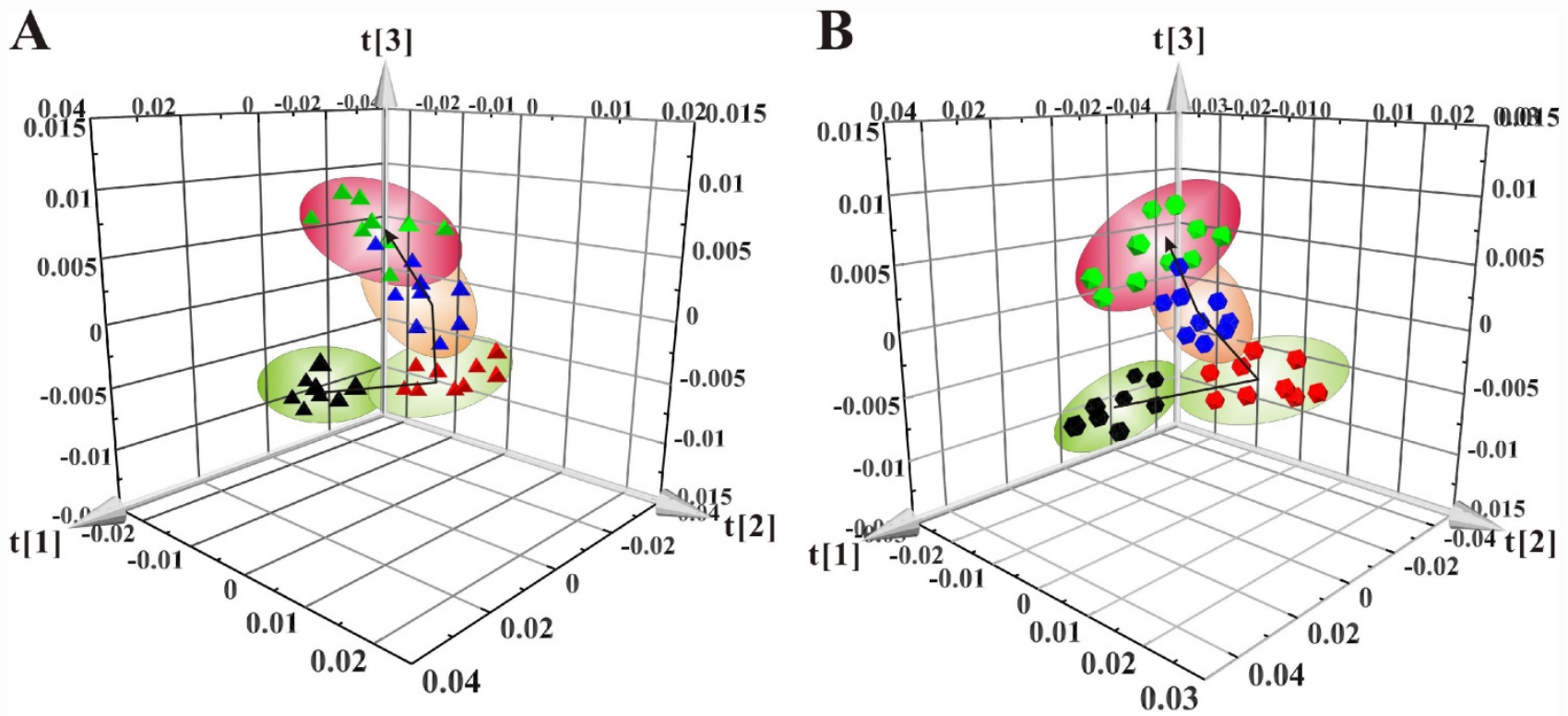

Figure 4. The principal component analyses of serum $1 \mathrm{H}$ NMR spectra data. (A) PCA of the LDLR ${ }^{-/-}$, PSGL-1 $1^{+/+}$mice at $8 w(\boldsymbol{\Delta}), 8+8 w(\boldsymbol{\Delta}), 8+12 w(\boldsymbol{\Delta})$ and $8+16 w$ $(\boldsymbol{\Delta})\left(\mathrm{R}^{2}=0.900, \mathrm{Q}^{2}=0.814\right) ;(\mathrm{B})$ PCA of LDLR $-/-$ PSGL-1-l- mice at $8 \mathrm{w}(\bullet), 8+8 \mathrm{w}(\bullet), 8+12 \mathrm{w}(\bullet)$ and $8+16 \mathrm{w}(\bullet)\left(\mathrm{R}^{2}=0.949, \mathrm{Q}^{2}=0.894\right)$. 

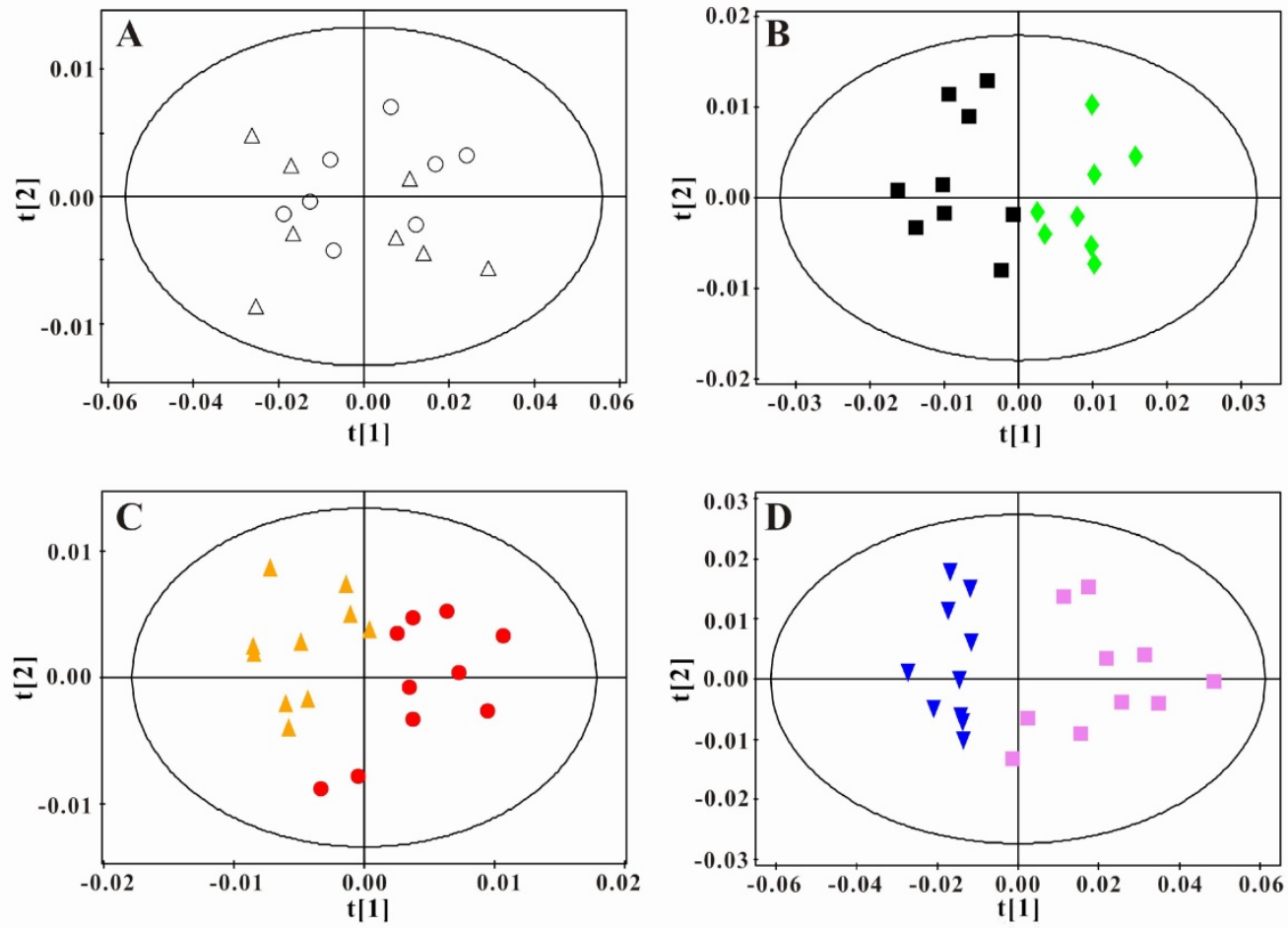

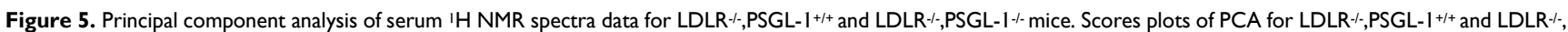

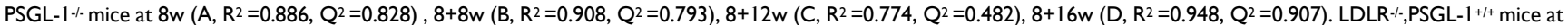
$8 \mathrm{w}(0), 8+8 \mathrm{w}(\boldsymbol{\bullet}), 8+12 \mathrm{w}(\bullet)$ and $8+16 \mathrm{w}(\boldsymbol{\nabla})$; LDLR -1, PSGL-1- mice at $8 w(\triangle), 8+8 w(\bullet), 8+12 \mathrm{w}(\boldsymbol{\Delta})$ and $8+16 \mathrm{w}(\boldsymbol{\bullet})$.

OPLS-DA models were established to find the metabolites differences induced by PSGL-1 deficiency with the progression of AS. The coefficients-coded line loadings were shown, which demonstrated the hot colored metabolites (e.g. red) with important contribution to the differentiation between

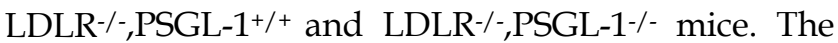
clear classifications between the two kinds of mice at $8+8 w, 8+12 w$ and $8+16 w$ were shown in the scores plots (Figure 6A1, B1 and $\mathrm{C} 1$ ). According to the histopathological evaluation, $8+8 w, 8+12 w$ and $8+16 \mathrm{w}$ were denoted as the early, medium and late stage of atherosclerosis. Compared with the LDLR $-/$, PSGL-1 ${ }^{+/+}$mice, the spectra in LDLR - ,PSGL-1\% mice at early stage of atherosclerosis showed higher levels of HDL, valine, alanine, acetate, methionine, pyruvate, choline, PC, GPC, taurine and glycine, and lower levels of LDL+VLDL and lactate (Figure 6A2). At the mid-term of AS, the levels of HDL, valine, alanine, methionine, choline, PC, GPC, taurine, glycine, LDL+VLDL and lactate maintained the same increase trend. Furthermore, the increased level of glutamine was found at the end of the $8+12 \mathrm{w}$ (Figure 6B2). At the late stage of AS, the changes of LDL+VLDL, glutamine, choline, taurine and glycine were same as the previous stages. Besides these metabolic changes, the increased levels of lactate and citrate were also observed at $8+16 \mathrm{w}$ (Figure $6 \mathrm{C} 2$ ). The metabolites accounting for the metabolic differences with statistical significance were summarized (Table 2). The levels of LDL+VLDL, HDL, lactate, valine, acetate, pyruvate, choline, PC/GPC and glycine showed statistical significance at the early stage of atherosclerosis, while lactate, citrate and glutamine also showed statistical significance at the late stage of atherosclerosis. These metabolites changes in LDLR $/$ ,PSGL-1\% mice were closely correlated with the regulation of the multiple biochemical pathways, including lipid and glucose metabolism, amino acid and phospholipid metabolism in LDLR ${ }^{-/}$mice (Figure 7).

\section{Discussion}

The LDLR $^{-/}$mice is a classic model of atherosclerosis in which the development of aorta plaque is similar to the clinic atherosclerosis progression. Compared with ApoE-/- mice, the lipoprotein spectrum of LDLR-/- mice is more similar to that of human, which is helpful to deduce the relationship between lipoprotein change and human atherosclerosis. It is important to analyze the lipoprotein change induced by drugs or food. LDLR/mice is beneficial to compare between the model feeding group and the normal group. For LDLR $/$ mice, plasma cholesterol is sensitive to cholesterol in the feeding and it is easy to control plasma cholesterol levels by changing the cholesterol content in the feeding diets, making the lesion closer to human 
disease. Moreover, the atherosclerosis plaque can develop to be fibrotic plaque or fibrous cap. Therefore, all the stages of human atherosclerosis can be simulated in LDLR $/$ - mice. Associating with the selectin, PSGL-1 could regulate leukocyte migration to inflammatory sites and mediate signal transmission [16, 37-38]. The previous study reported that PSGL-1 deletion directly affected the adhesion of leukocytes and endothelial cells and indirectly reduced atherosclerosis in $\mathrm{ApoE}^{-/}$mice by modulating endothelial cell factor [17]. It was shown that the AS plaque in ApoE-/- mice with 8 weeks Western diet were found to be alleviated. However, the effects of PSGL-1 deficiency on the AS development and progression and the metabolic regulation were not performed. In this study, the pathological results showed that PSGL-1 deficiency reduced the plaque area, the inflammatory infiltration and fiber hyperplasia, and the development of AS was inhibited in LDLR $/$, $P S G L-1 \%$ mice. Moreover, the PSGL-1 deficiency regulated the dyslipidemia and serum metabolism. It was showed that LDL, VLDL, HDL, lactate, valine, acetate, pyruvate, choline, PC, GPC and glycine significantly changed at the early stage of atherosclerosis, while lactate, citrate and glutamine showed statistical significance at the late stage of atherosclerosis. The results showed that the PSGL-1 deficiency inhibited the progression of AS and regulated the metabolic disorders in LDLR-/mice.

\section{PSGL-1 deficiency regulates lipids and phospholipids metabolism}

Dyslipidemia, also known as hyperlipidemia, is recognized as a causative determinant of coronary artery disease and atherosclerotic cardiovascular disease [39-40]. It is characterized by increase of TC, TG, and LDL-C in conjunction with decrease of HDL-C and is normally caused by abnormal lipid metabolism [41]. In this study, the biochemical assessment of blood showed that TC, LDL-C and TG were significantly increased with atherosclerosis aggression in LDLR $^{-/}, \mathrm{PSGL}^{-1 /+}{ }^{++}$mice, which is consistent with the previous observations [20,29,31]. It was noted that PSGL-1 deficiency greatly reduced the levels of TG, TC and LDL-C, accompanied by increased HDL-C. The metabonomics study also showed the similar changes in LDL and HDL, which was a possible cause responsible for the inhibiting the AS development by PSGL-1 deficiency.
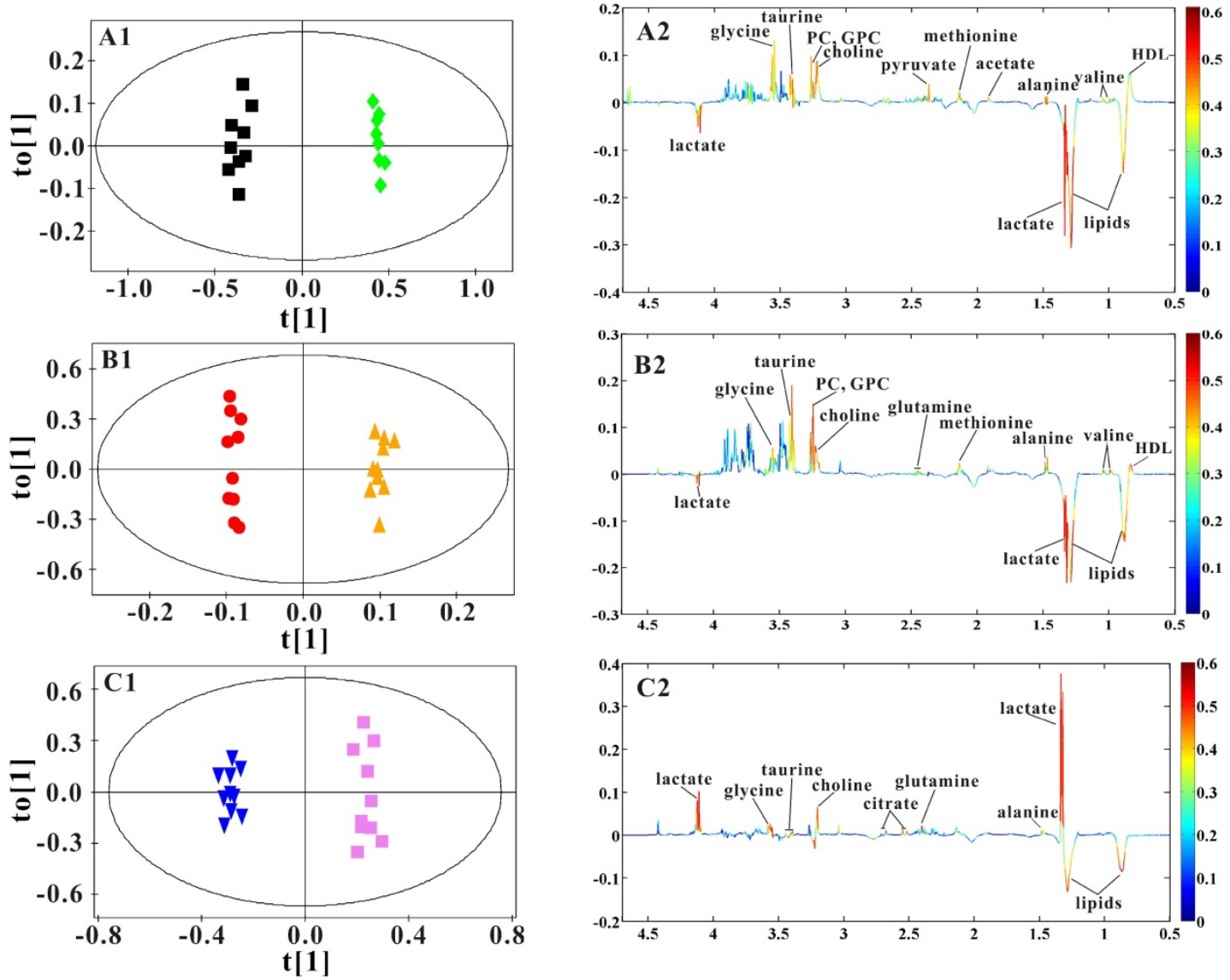

Figure 6. OPLS-DA results of serum $1 \mathrm{H}$ NMR spectra data for LDLR ${ }^{-/,}, \mathrm{PSGL}-1^{+/+}$and LDLR ${ }^{-/,}, \mathrm{PSGL}-1^{-/-}$mice. Scores plots $(\mathrm{A} 1, \mathrm{~B} 1, \mathrm{Cl})$ and coefficient-coded loadings plots $(\mathrm{A} 2$, $B 2, C 2)$ from serum 'H spectra data at $8+8 w\left(R^{2} X=0.681, Q^{2} Y=0.949, Q^{2}=0.801\right), 8+12 w\left(R^{2} X=0.831, Q^{2} Y=0.996, Q^{2}=0.669\right)$ and $8+16 w\left(R^{2} X=0.878, Q^{2} Y=0.987, Q^{2}=0.817\right)$.

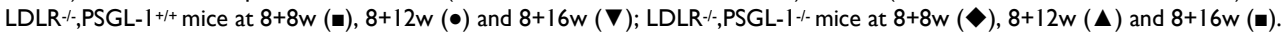




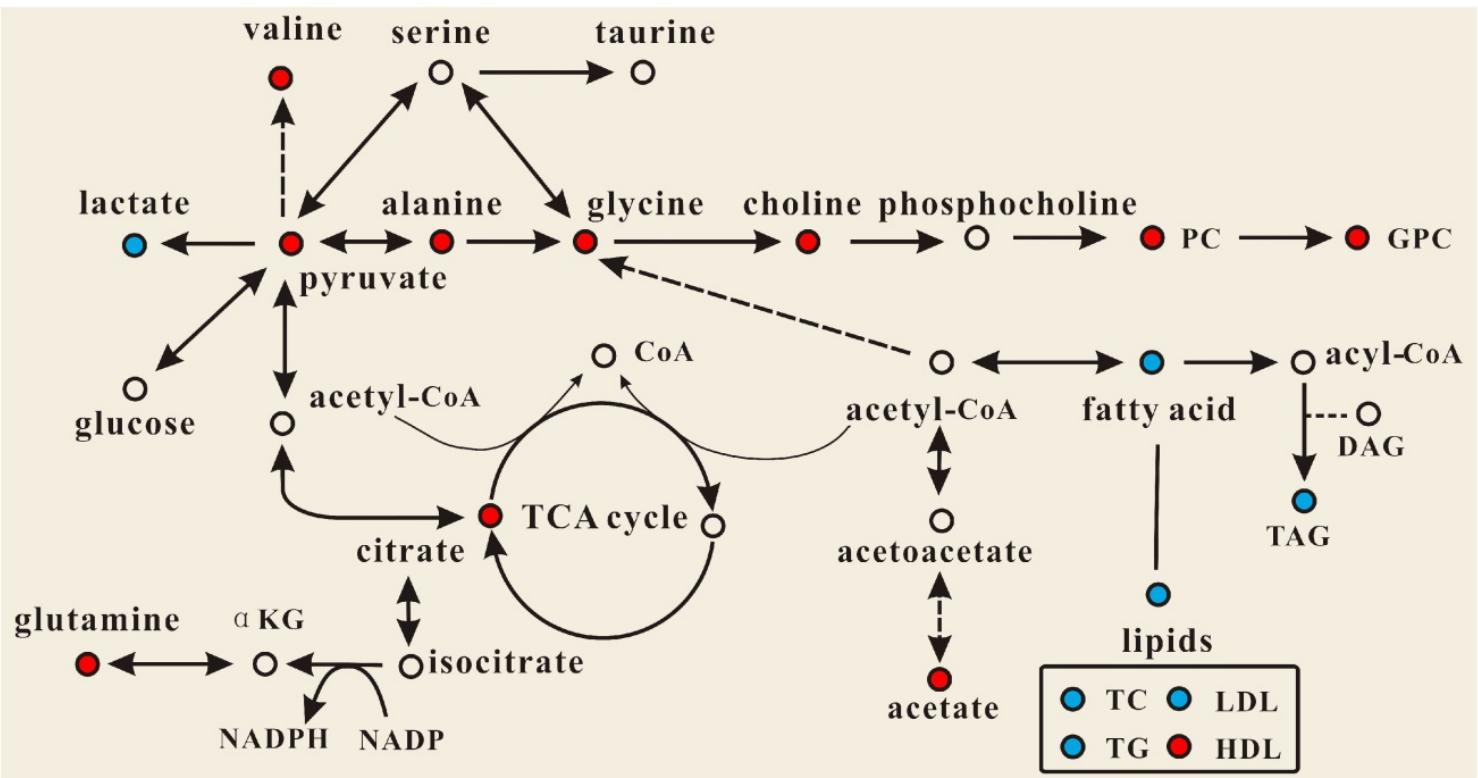

Figure 7. Effect of PSGL-1 deficiency on the serum metabolic pathways involved in LDLR-/- mice. The blue or red metabolites suggest a lower or higher level in LDLR -1, ,PSGL-1- mice compared with the LDLR -1, PSGL-1+/+, respectively.

Table 2. Statistical analysis results of serum metabolites in LDLR ${ }^{-/,}, \mathrm{PSGL}^{-1^{+/+}}$and $\mathrm{LDLR}^{-/-}, \mathrm{PSGL-1/-}$ mice.

\begin{tabular}{|c|c|c|c|c|c|}
\hline$\delta 1 \mathrm{H}(\mathrm{ppm})^{\mathrm{a}}$ & Metabolites & Pathway & $8+8 w$ & $8+12 w$ & $8+16 w$ \\
\hline $0.80-0.84$ & HDL & Lipid metabolism & $\uparrow * *$ & $\uparrow \uparrow$ & - \\
\hline $0.84-0.88(\mathrm{~m}), 1.20-1.30(\mathrm{~m})$ & $\mathrm{LDL}+\mathrm{VLDL}$ & Lipid metabolism & $\downarrow^{* * *}$ & $\downarrow^{*}$ & $\downarrow^{* *}$ \\
\hline $1.00,1.03(\mathrm{~d})$ & valine & Amino acid metabolism & $\uparrow^{*}$ & $\uparrow$ & - \\
\hline $1.33(\mathrm{~d}), 4.11(\mathrm{t})$ & lactate & Carbohydrate metabolism & $\downarrow^{*}$ & $\downarrow$ & $\uparrow^{*}$ \\
\hline $1.48(\mathrm{~d})$ & alanine & Amino acid metabolism & $\uparrow$ & $\uparrow$ & $\uparrow$ \\
\hline $1.91(\mathrm{~s})$ & acetate & Carbohydrate metabolism & $\uparrow^{*}$ & - & - \\
\hline $2.14(\mathrm{~s})$ & methionine & Amino acid metabolism & $\uparrow$ & $\uparrow$ & - \\
\hline $2.12(\mathrm{~m}), 2.44(\mathrm{~m})$ & glutamine & Amino acid metabolism & - & $\uparrow$ & $\uparrow^{*}$ \\
\hline $2.37(\mathrm{~s})$ & pyruvate & Carbohydrate metabolism & $\uparrow * *$ & - & - \\
\hline $2.56,2.65(\mathrm{dd})$ & citrate & Carbohydrate metabolism & - & - & $\uparrow^{*}$ \\
\hline $3.20(\mathrm{~s})$ & choline & Lipid metabolism & $\uparrow * * *$ & $\uparrow^{* *}$ & $\uparrow$ \\
\hline $3.22(\mathrm{~s}), 3.24(\mathrm{~s})$ & PC and GPC & Lipid metabolism & $\uparrow^{*}$ & $\uparrow * *$ & - \\
\hline $3.43(\mathrm{t})$ & taurine & Amino acid metabolism & $\uparrow$ & $\uparrow$ & $\uparrow$ \\
\hline $3.57(\mathrm{~s})$ & glycine & Amino acid metabolism & $\uparrow * * *$ & $\uparrow$ & $\uparrow$ \\
\hline
\end{tabular}

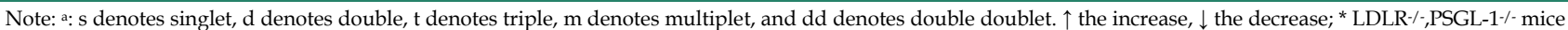
compared with LDLR $\%$,PSGL- $1^{+/+}$mice at $8 \mathrm{w}, 8+8 \mathrm{w}, 8+12 \mathrm{w}$ and $8+16 \mathrm{w} .{ }^{*} \mathrm{p}<0.05,{ }^{* *} \mathrm{p}<0.01,{ }^{* * *} \mathrm{p}<0.001$.

Studies have reported that the levels of choline, $P C$ and GPC were decreased in LDLR ${ }^{-/-}$mice $[20,31]$. In this study, we found that the LDLR $^{-/}$, PSGL-1/mice had higher levels of choline, PC and GPC, indicating PSGL-1 deficiency regulated the phospholipid disorders in atherosclerosis procession of LDLR $\%$ mice. Choline has an essential role in several biological processes including cell signaling, vitamins metabolism, lipid transport and metabolism [42]. Choline-deficiency is now thought to be a factor for some diseases such as liver disease, atherosclerosis and neurological disorders [43]. Moreover, Olthof [44] suggested that choline intake could decrease the cardiovascular disease risk induced by excessive homocysteine. The study reported that the inflammatory factors of C-reactive protein, TNF- $a$ and IL6 reduced in the subjects whose diets were rich in choline and betaine [45]. Rajabi A [46] showed that choline supplementation could improve liver health by keeping cholesterol homeostasis in Pemt/LDLR knockout mice administered with a high-fat diet. Therefore, the up-regulation of choline by PSGL-1 deficiency was helpful for inhibiting the AS progression in LDLR $-/-$,PSGL-1/- mice.

PC and GPC are important components of phospholipids that constitute a cell membrane double layer structure to ensure the stability of the cell metabolism [47]. A study reported that choline, PC and GPC significantly decreased in LDLR $/$-mice [20], which indicated that there were disorders of cell membrane metabolism during atherosclerosis progression. In this study, PC and GPC were up-regulated, which suggested the deficiency of PSGL-1, as a type 1 membrane glycoprotein, affected 
the cell membrane metabolism and further reduced atherosclerosis.

\section{PSGL-1 deficiency regulates energy metabolism}

ATP is required for energy generation in the body, which mainly comes from glycolysis, lipid oxidation and glucose metabolism. In this study, compared with LDLR $^{-/-, P S G L-1 /++}$ mice, we found that the level of lactate (a product of glycolysis) was decreased at the early and medium stages in PSGL-1\%,LDLR $\%$ mice. In addition, a few of metabolites involved in TCA cycle were increased in PSGL-1/-,LDLR $/$ - mice, including acetate and citrate. These metabolic changes showed the down-regulated glycolysis and activated TCA induced by PSGL-1 deficiency. Previous researches reported that energy metabolism was perturbed in the animal models and clinical studies of atherosclerosis [31, 48]. Atherosclerotic lesions contained the regions of hypoxia, and the hypoxia promoted glycolysis and lactate accumulation $[49,50]$, while PSGL-1 deficiency reduced the AS lesions and partly inhibited the glycolysis. However, lactate was up-regulated at the late stage of AS in PSGL-1/-,LDLR $\%$ - mice, which was a possible result of increased need of energy with the AS progression.

TCA cycle, as a center of the regulation of cell metabolism and energy homeostasis, oxidizes and decomposes fatty acids, amino acids and carbohydrates [51-52]. Acetate and citrate are important intermediate products in TCA cycle. Pyruvate is an important chemical compound in energy metabolism. When oxygen is present, it can supplies energy to cells through the TCA cycle, and ferments to produce lactate at oxygen is lacking. The increased pyruvate, acetate and citrate in PSGL-1 $\%$, LDLR $\%$ mice indicated the activated TCA cycle and energy metabolism.

\section{PSGL-1 deficiency regulates amino acid metabolism}

With the development of atherosclerosis, PSGL-1 deficiency induced the changes of amino acid metabolism, including the increased glycine, valine, glutamine and alanine. Glycine is useful for the effects of anti-oxidative and anti-inflammatory [53] and it is negatively correlated with the cardiovascular diseases such as obesity, hypertension, etc. [54-55]. Ding Y and colleagues [56] performed the follow-up survey of 4109 participants and found that plasma glycine in angina patients with acute myocardial infarction risk was reduced. Therefore, the increased glycine in PSGL-1 $\%$,LDLR $\%$ mice was correlated with the reduced AS lesions, which was consistent with the previous studies [54-56]. Glutamine was synthesized in the body by pyruvate metabolism, which assisted glucose metabolism and improved body energy. Fujimoto et al [57] found that the alanine allele protected macrophages against the oxLDL-induced apoptosis and reduced the risk of cardiovascular diseases. In addition, a study has shown that the $\mathrm{LDLR}^{-/}$pregnant mice exhibited significantly decreased valine [58]. Martin-Lorenzo M [59] also found the lower levels of valine in atherosclerotic arteries, and it was linked to cardiovascular risk. These changes of amino acids associated with the inhibition of atherosclerosis demonstrated the metabolic regulation of PSGL-1 deficiency in LDLR $\%$-,PSGL-1 $\%$ mice.

\section{Conclusions}

In this work, we found that the deficiency of PSGL-1 inhibited the development and progression of AS in the LDLR $/$ mice, and it regulated the serum metabolic profiles, mainly involving glucose metabolism, lipid metabolism, amino acid and phospholipid metabolism. This study provided a metabolic research basis for the PSGL-1 effects on atherosclerosis.

\section{Abbreviations}

AS, atherosclerosis; LDLR, low density lipoprotein receptor; PSGL-1, P-selectin glycoprotein ligand-1; NMR, nuclear magnetic resonance; CPMG, Carr-Purcell- Meiboom-Gill; FIDs, free-induction decays; PCA, principal component analysis; OPLS-DA, orthogonal partial least-squares discriminant analysis; TC, total cholesterol; TG, Triglycerides; LDL-C, low-density lipoprotein cholesterol; HDL-C, high-density lipoprotein cholesterol; LDL, low-density lipoprotein; VLDL, very low-density lipoprotein; PC, phosphorylcholine; GPC, glycerophosphorylcholine; HE stain, hematoxylin and eosin stain; ATP, adenosine triphosphate; TCA cycle, tricarboxylic acid cycle.

\section{Acknowledgements}

The study was supported by the Science and Technology Planning Projects of Guangdong Province (No. 2016A030303062, 2015A020211033).

\section{Competing Interests}

The authors have declared that no competing interest exists.

\section{References}

[1] Libby P, Ridker PM. Progress and challenges in translating the biology of atherosclerosis. Nature. 2011; 473: 317-325.

[2] Lusis AJ. Atherosclerosis. Nature.2000; 407(6801): 233-241. 
[3] Rader DJ. Translating molecular discoveries into new therapies for atherosclerosis. Nature.2008; 451: 904-913.

[4] Vacek TP, Rehman S, Neamtu D, et al. Matrix metalloproteinases in atherosclerosis: role of nitric oxide, hydrogen sulfide, homocysteine, and polymorphisms. Vasc Health Risk Managm.2015; 11: 173-183.

[5] Wang M, Kim SH, Monticone RE, et al. Matrix metalloproteinases promote arterial remodeling in aging, hypertension, and atherosclerosis. Hypertension. 2015; 65(4): 698-703.

[6] Dimas G, Iliadis F, Grekas D. Matrix metalloproteinases, atherosclerosis, proteinuria and kidney disease: Linkage-based approaches. Hippokratia. 2013; 17(4): 292-297.

[7] Bonomini F, Tengattini S, Fabiano A, et al. Atherosclerosis and oxidative stress. Histol Histopathol. 2008; 23(3): 381-390.

[8] Greaves D R, Channon K M. Inflammation and immune responses in atherosclerosis. Trends Immunol. 2002; 23(11): 535-541.

[9] Vanassche T, Huygelen V, Crabtree M J, et al. Gene therapy targeting inflammation in atherosclerosis. Curr Pharm Des. 2011;17(37): 4210-4223.

[10] Ozkan C, Altinova AE, Cerit ET, et al. Markers of early atherosclerosis, oxidative stress and inflammation in patients with acromegaly. Pituitary. 2015;18(5): 621-629.

[11] Lloyd-Jones D M. Cardiovascular risk prediction: basic concepts, current status, and future directions. Circulation. 2010;121(15): 1768-1777.

[12] Musunuru K. Atherogenic dyslipidemia: cardiovascular risk and dietary intervention. Lipids. 2010;45(10): 907-914.

[13] Chen H, Chen L, Tang D D, et al. Metabolomics Reveals Hyperlipidemic Biomarkers and Antihyperlipidemic Effect of Poria cocos. Current Metabolomics.2016,4(2):104-115.

[14] McEver RP, Cummings RD. Perspectives series: cell adhesion in vascular biology. Role of PSGL-1 binding to selectins in leukocyte recruitment. J Clin Invest.1997; 100(3): 485-491.

[15] Vachino G, Chang XJ, Veldman GM, et al. P-selectin glycoprotein ligand-1 is the major counter-receptor for P-selectin on stimulated T cells and is widely distributed in non-functional form on many lymphocytic cells. J Boil Chem.1995; 270(37): 21966-21974.

[16] Wang HB, Wang JT, Geng JG, et al. P-selectin primes leukocyte integrin activation during inflammation. Nat Immunol. 2007; 8(8): 882-892.

[17] Luo W, Wang H, Ohman MK, et al. P-selectin Glycoprotein Ligand-1 Deficiency Leads to Cytokine Resistance and Protection against Atherosclerosis in Apolipoprotein E Deficient Mice. Atherosclerosis. 2012; 220(1): 110-117.

[18] Li D, Liu Y, Zhang X, et al. Inhibition of soluble epoxide hydrolase alleviated atherosclerosis by reducing monocyte infiltration in $\mathrm{Ldlr}(-/)$ mice. J Mol Cell Cardiol. 2016;98: 128-137.

[19] Ishibashi S, Goldstein JL, Brown MS, et al. Massive xanthomatosis and atherosclerosis in cholesterol-fed low density lipoprotein receptor-negative mice. J Clin. Invest.1994; 93(5): 1885-1893.

[20] Li D, Zhang L, Dong F, et al. Metabonomic Changes Associated with Atherosclerosis Progression for LDLR - Mice. J Proteome Res. 2015; 14(5): 2237-2254.

[21] Nicholson JK, Lindon JC. 'Metabonomics': understanding the metabolic responses of living systems to pathophysiological stimuli via multivariate statistical analysis of biological NMR spectroscopic data. Xenobiotica.1999; 29(11): 1181-1189.

[22] Holmes E, Wilson ID. Metabolic phenotyping in health and disease. Cell. 2008; 134(5): 714-717.

[23] Miao H, Li MH, Zhang X, et al. The antihyperlipidemic effect of Fu-Ling-Pi is associated with abnormal fatty acid metabolism as assessed by UPLC-HDMS-based lipidomics. Rsc Advances. 2015, 5(79):64208-64219.

[24] Holmes E, Tang H, Wang $Y$, et al. The assessment of plant metabolite profiles by NMR-based methodologies. Planta Med. 2006; 72: 771-785.

[25] Chen H, Miao H, Feng Y L, et al. Metabolomics in dyslipidemia.[J]. Adv Clin Chem, 2014, 66: 101-119.

[26] Kaddurahdaouk R, Bogdanov M B, Wikoff W R, et al. Pharmaco metabolomic mapping of early biochemical changes induced by sertraline and placebo. Translational Psychiatry. 2013; 3: e223.

[27] Yang Y, Wang L, Wang S, et al. Study of metabonomic profiles of human esophageal carcinoma by use of high-resolution magic-angle spinning ${ }^{1} \mathrm{H}$ NMR spectroscopy and multivariate data analysis. Analytical and bioanalytical chemistry. 2013; 405(10): 3381-3389.

[28] Zhang X, Wang Y, Hao F, et al. Human serum metabonomic analysis reveals progression axes for glucose intolerance and insulin resistance statuses. Journal of proteome research. 2009; 8(11): 5188-5195.

[29] Miao H, Chen H, Pei S, et al. Plasma lipidomics reveal profound perturbation of glycerophospholipids, fatty acids, and sphingolipids in diet-induced hyperlipidemia. Chemico-Biological Interactions. 2015; 228:79-87.

[30] Guo W, Jiang C, Yang L, et al. Quantitative Metabolomic Profiling of Plasma, Urine, and Liver Extracts by (1)H NMR Spectroscopy Characterizes Different Stages of Atherosclerosis in Hamsters. Journal of proteome research. 2016; 15(10): 3500-3510.

[31] Yang Y, Liu Y, Zheng L, et al. Serum metabonomic analysis of apoE(-/-) mice reveals progression axes for atherosclerosis based on NMR spectroscopy. Molecular bioSystems. 2014;10(12): 3170-3178.

[32] Zhao YY, Cheng XL. Lipidomics applications for discovering biomarkers of diseases in clinical chemistry. International review of cell and molecular biology. 2014; 313: 1-26.
[33] Trygg, Johan, Wold, Svante. Orthogonal projections to latent structures (O-PLS). Journal of Chemometrics. 2002; 16(3): 119-128.

[34] Lennart E, Johan T, Svante W. CV-ANOVA for significance testing of PLS and OPLS $®$ models. Journal of Chemometrics. 2010; 22(11-12): 594-600.

[35] Liao P, Wei L, Wu H, et al. Biochemical effects of gadolinium chloride in rats liver and kidney by 1H NMR metabolomics . Journal of Rare Earths. 2009; 27 (02): 280-287.

[36] Wang Y, Zhang L, Chen W L, et al. Rapid Diagnosis and Prognosis of de novo Acute Myeloid Leukemia by Serum Metabonomic Analysis. Journal of Proteome Research. 2013;12(10):4393-4401.

[37] Puegge J, Wang Y, Roller J, et al. Adhesive Mechanisms of Histone-Induced Neutrophil-Endo -thelium Interactions in the MuscleMicrocirculation. Eur Surg Res. 2016; 56(1-2): 19-31.

[38] Stadtmann A, Germena G, Block H, et al. The PSGL-1-L-selectin signaling complex regulates neutrophil adhesion under flow. J Exp Med. 2013; 210(11): 2171-2180.

[39] Teslovich TM, Musunuru $K$, Smith AV, et al. Biological, clinical and population relevance of 95 loci for blood lipids. Nature. 2010; 466(7307): 707-713.

[40] LaRosa J C. Prevention and treatment of coronary heart disease: who benefits? Circulation. 2001; 104(14):1688-1692.

[41] Miao H, Zhao Y H, Zhao Y Y, et al. Lipidomics biomarkers of diet-induced hyperlipidemia and its treatment with Poria cocos.[J]. Journal of Agricultural \& Food Chemistry.2016; 64(4):969-979.

[42] Blusztajn JK, Slack BE. Neuroprotective Actions of Dietary Choline. Nutrients. 2017;9(8).

[43] Zeisel SH. Choline: an essential nutrient for public health. Nutrition reviews. 2009; 67(11): 615-623.

[44] Olthof MR, Brink EJ, Katan MB. Choline supplemented as phosphatidylcholine decreases fasting and postmethionine-loading plasma homocysteine concentrations in healthy men. Am J Clin Nutr. 2005;82(1): 111-117.

[45] Detopoulou P, Panagiotakos DB, Antonopoulou S, et al. Dietary choline and betaine intakes in relation to concentrations of inflammatory markers in healthy adults: the ATTICA study. Am J Clin Nutr. 2008; 87(2): 424-430.

[46] Rajabi A, Castro GS, Da Silva RP, et al. Choline supplementation protects against liver damage by normalizing cholesterol metabolism in Pemt/Ldlr knockout mice fed a high-fat diet. The Journal of nutrition. 2014; 144(3): 252-257.

[47] Mestdagh R, Dumas ME, Rezzi S, et al. Gut microbiota modulate the metabolism of brown adipose tissue in mice. J.Proteome Res. 2012; 11(2): 620-630.

[48] Soininen P, Kangas AJ, Würtz P, et al. Quantitative serum nuclear magnetic resonance metabolomics in cardiovascular epidemiology and genetics. Circulation. Cardiovascular genetics. 2015; 8(1): 192-206.

[49] Sluimer JC, Gasc JM, Van Wanroij JL, et al. Hypoxia, hypoxia-inducible transcription factor, and macrophages in human atherosclerotic plaques are correlated with intraplaque angiogenesis. J Am Coll Cardiol. 2008; 51(13): 1258-1265.

[50] Hultén LM. The role of hypoxia in atherosclerosis. Current opinion in lipidology. 2009; 20(5): 409-414

[51] He W, Miao FJ, Lin DC, et al. Citric acid cycle intermediates as ligands for orphan G-protein-coupled receptors. Nature. 2004; 429(6988): 188-193.

[52] Ishizaka S, Kikuchi E, Higashino T, et al. Effects of acetate on the immune system of mice. Int J Immunopharmacol. 1990; 12 (1) : 135-143.

[53] Senthilkumar R, Sengottuvelan M, Nalini N, et al. Protective effect of glycine supplementation on the levels of lipid peroxidation and antioxidant enzymes in the erythrocyte of rats with alcohol-induced liver injury. Cell Biochem Funct. 2004; 22: 123-128.

[54] Oberbach A, Bluher M, Wirth $\mathrm{H}$, et al. Combined proteomic and metabolomic profiling of serum reveals association of the complement system with obesity and identifies novel markers of body fat mass changes. J Proteome Res. 2011; 10: $4769-4788$.

[55] EI H M, Perez I, Baños G., et al. Is glycine effective against elevated blood pressure. Curr Opin Clin Nutr Metab Care. 2006; 9: 26-31.

[56] Ding Y , Svingen GF , Pedersen ER, et al. Plasma Glycine and Risk of Acute Myocardial Infarction in Patients With Suspected Stable Angina Pectoris. J Am Heart Assoc. 2015;5(1): e002621.

[57] Fujimoto H, Taguchi J, Imai $Y$, et al. Manganese superoxide dismutase polymorphism affects the oxidized low-density lipoprotein-induced apoptosis of macrophages and coronary artery disease. European heart journal. 2008; 29(10): 1267-1274.

[58] Bhasin KK, Van Nas A, Martin LJ, et al. Maternal low-protein diet or hypercholesterolemia reduces circulating essential amino acids and leads to intrauterine growth restriction. Diabetes. 2009; 58(3): 559-566.

[59] Martin-Lorenzo M, Gonzalez-Calero L, Maroto A S, et al. Cytoskeleton deregulation and impairment in amino acids and energy metabolism in early atherosclerosis at aortic tissue with reflection in plasma. Biochimicaet biophysica acta. 2016; 1862(4): 725-732. 